\title{
Study of interdigital electrode structures for the detection of water spray
}

\author{
Jakob Döring, Lakshan Tharmakularajah, Karl-Ludwig Krieger \\ Institute of Electrodynamics and Microelectronics (ITEM.ae), University of Bremen \\ \{doering, lakshan, krieger\}@item.uni-bremen.de
}

\begin{abstract}
This paper presents a study of interdigital electrode structures suitable for the detection of road surface wetness. The structures are studied by means of the finite element method (FEM) with the aim of analyzing the effect of varying design parameters on the detection of water. A variation of the number of digits on a fixed electrode area shows a trade-off between dynamic range and penetration depth. Furthermore, increasing the ratio of electrode width and distance results in an enhanced signal-to-noise ratio. In addition, the effects of varying the substrate thickness and applying a shield electrode are evaluated. The study of placing a coating layer on the electrode structure shows the significance of the choice of its thickness and permittivity. Finally, simulations with different water drop sizes show the usability of detecting even small amounts of drops and verify the assumptions made before.
\end{abstract}

Keywords: Planar capacitive sensors, interdigital transducers, wetness detection, vehicle safety systems, finite element method.

\section{Introduction}

Even nowadays, in times of steadily increasing sensor functionality and related driver assistance functions, there are numerous traffic accidents involving personal injury every year. In 2017 , there were 302,656 of such accidents in Germany in which a total of 393,492 people were injured [1]. In around $5 \%$ of these cases, the cause of the accident is stated to be the road surface condition. Although today's vehicles are already equipped with extensive environment sensors, including radar, lidar, ultrasound and camera systems, these do not provide direct information about a road's current state. Currently, the driver of the vehicle estimates the current state intuitively and experience-based and adapts his driving accordingly. Misjudgments can have fatal consequences for the driver and other traffic participants.

With the increasing level of automation in today's motor vehicles, the detection of road surface conditions is continuing to increase in importance. Particularly with regard to highly or fully automated driving (SAE Level 4 and 5), knowledge of the current condition is of great relevance in order to be able to handle safety-critical driving situations due to weather conditions without the visual control of the driver. In addition to existing data from environment and motor vehicle sensors, innovative approaches are needed to reliably monitor road surface condition. Thus, the driver or the system can be warned in time of potentially dangerous situations that could lead to an unstable vehicle condition due to loss of traction.

A road surface condition that can lead to a significant loss of traction is road wetness. In our previous work [2], we presented a novel approach for detecting road surface wetness, which can prospectively contribute to increase the safety of vehicle passengers and traffic participants in times of highly or fully automated driving. Here, planar capacitive sensors are placed on a motor vehicle's wheel arch liner. If the road surface is wet, the tires cause water to whirl up, which impinges upon the wheel arch liner in the form of drops (water spray). The capacitive sensor can detect the water drops and thus indirectly infer a wet road surface. Since a greater water film height leads to more water being whirled up (to a certain extent) and thus more drops impinging on the sensor, a basic distinction between wetness levels is possible.

As the focus in [2] was on demonstrating the feasibility of the approach, a limited design space was used for the simulations. Furthermore, effects on varying design parameters were neglected. In this paper, we present an enhanced study of interdigital electrode structures for the detection of water spray. The structures are studied by means of the finite element method (FEM) in COMSOL Multiphysics. Fundamental characteristics of interdigital electrode structures, including electrode width and distance, are initially examined by assuming a static water layer. Furthermore, the transducer's substrate thickness and the effect of a shield 
electrode are studied. Since the main application is road wetness detection, a coating layer is needed to mechanically protect the electrodes. For this reason, the influence of a coating layer is studied in the simulations. Finally, we simulate selected electrode structures with water drops of different size to show the assumptions previously made for a static water layer hold.

\section{Planar capacitive sensors}

Due to its wide range of features, a possible type of sensor suitable for the detection of road surface wetness is the capacitive sensor, as shown in [2]. Because of desirable features like robustness, low costs and flexibility in electrode design [3, 4, 5] they have already been used in a wide range of applications. For instance, they are used for liquid level sensing in containers [6], the detection of ice in liquid conveyor pipes [7] or the measurement of soil water content [8].

The measuring principle of capacitive sensors is based on the interaction of a monitored medium and an applied electric field between the sensor electrodes $[3,9]$. Since the electric field penetrates through the medium, electric displacement is generated inside it, which alters the charge stored between the sensor electrodes. Thus, the sensor capacitance changes, allowing inferences to be drawn about the permittivity of the medium. Hence, the determination of system parameters that correlate with the permittivity of the medium (e.g. moisture) is possible.

The more conventional parallel-plate capacitive sensor is based on the same principle of operation as a planar capacitive sensor [10]. Between the two parallel plates, one representing the driving electrode and the other representing the sensing electrode, an electric field is distributed uniformly [3]. Due to the much larger dimensions of the electrode surface compared to the electrode thickness, the fringe field is negligible. Since the electrodes are opened up in case of a planar capacitive sensor, the fringe field becomes predominant, which is why this kind of sensor is often denoted as a fringe field sensor in literature $[3,10,11]$. Even in the case of the planar capacitor, the electric field lines penetrate into the monitored medium. Thus, the sensor enables single-sided access to the medium, which is one of the most important advantages of a planar capacitive sensor in addition to the simple and low-cost fabrication process [9].

In order to meet the requirements of an application, in this case the detection of water spray, the choice of sensor geometry is critical as it is a major determination factor for sensor performance [12]. A suitable design of a planar capacitive sensor is an interdigital electrode structure, since the contribution of the fringing field effect in sensor capacitance is maximized $[8,11]$.
An interdigital electrode structure consists of two electrodes, one being the sensing electrode and the other being the driving electrode. Both electrodes are generally built up by the same number of digits $n$, which are separated by a distance $d$. Another parameter commonly used in the context of interdigital electrode structures is the spatial wavelength $\lambda$ [10]. It can be defined as the distance between the centerlines of adjacent digits belonging to the same electrode

$$
\lambda=2 \cdot(w+d) .
$$

In order to design an interdigital sensor, $\lambda$ has to be considered since the penetration depth $\gamma_{97 \%}$ of the electric field is approximately proportional to it [10]. There is no unique definition for the penetration depth in literature, but one possible way to evaluate it, is to study how deep the electric field penetrates into a monitored medium [9]. For this purpose, the thickness of the monitored medium is increased incrementally until the point is reached, where $97 \%$ of the asymptotic capacitance is obtained

$$
\frac{C\left(t_{\mathrm{m}}=\gamma_{97 \%}\right)-C\left(t_{\mathrm{m}}=0\right)}{C\left(t_{\mathrm{m}}=\infty\right)-C\left(t_{\mathrm{m}}=0\right)} \cdot 100 \%=97 \% \text {. }
$$

$C\left(t_{\mathrm{m}}=\gamma_{97 \%}\right)$ is the capacitance, where the thickness of the monitored medium $t_{\mathrm{m}}$ equals the penetration depth $\gamma_{97 \%}, C\left(t_{\mathrm{m}}=\infty\right)$ is the asymptotic capacitance for the monitored medium and $C\left(t_{\mathrm{m}}=0\right)$ the capacitance in the absence of a medium.

In addition to the penetration depth, there are further parameters usually used to evaluate interdigital electrode structures including signal strength, dynamic range and measurement sensitivity $[3,12]$. While the signal strength describes the output capacitance of a sensor for a monitored medium of a certain thickness $C\left(t_{\mathrm{m}}\right)$, the dynamic range $D R$ indicates the difference of the maximum capacitance for a monitored medium and the basic capacitance $B C$ (absence of a medium)

$$
D R=C\left(t_{\mathrm{m}}=\infty\right)-C\left(t_{\mathrm{m}}=0\right) .
$$

By maximizing the dynamic range, and simultaneously minimizing the basic capacitance, a maximum signal-to-noise ratio can be achieved. The measurement sensitivity $S$ can be defined as the ratio of the change in the sensor output to the change in the system variable. In the context of this paper, the thickness of the monitored medium represents the system variable. Thus, the sensitivity can be calculated from the ratio of changes in capacitance to changes of the medium's thickness

$$
S=\frac{\mathrm{d} C\left(t_{\mathrm{m}}\right)}{\mathrm{d} t_{\mathrm{m}}} .
$$




\section{FEM simulations}

The analytical modeling of interdigital electrode structures is not trivial compared to classic electrode shapes such as parallel-plate electrodes in which the electric field is distributed homogeneously between the plates $[8,10]$. For that reason, FEM is commonly used for sensor modeling, optimization and performance evaluation [12]. In order to study interdigital electrode structures and their design parameters with regard to the detection of water, we use COMSOL Multiphysics for two- and three-dimensional electrostatic FEM simulations.

Since the target application is the detection of water spray on a vehicle's wheel arch liner, we have limitations in design parameters, which are considered in the simulations, as for example the electrode area is fixed at $50 \mathrm{~mm} \times 50 \mathrm{~mm}$. Furthermore, we focus on printed circuit board (PCB) technology as it enables to fabricate small-scale units with high precision at low per-unit costs $[3,8,11]$. The relevant parameters and dimensions studied in the simulations are depicted in Fig. 1.

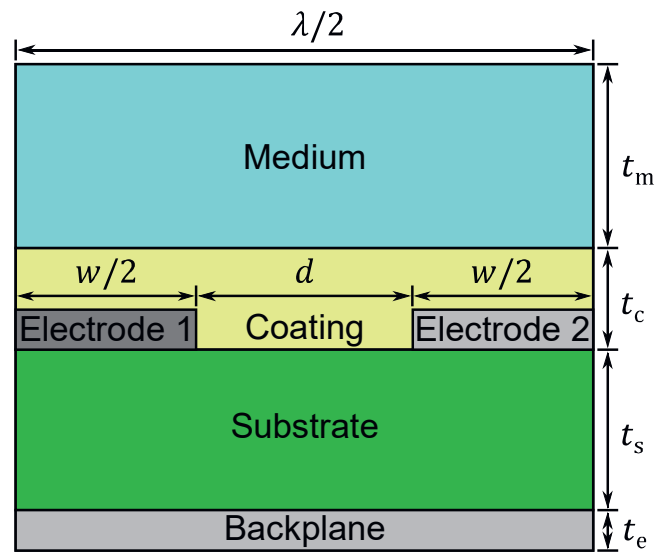

Fig. 1: Schematic cross-section view of an interdigital electrode structure with its relevant parameters and dimensions.

In general, we assume a static water layer thickness $t_{\mathrm{m}}$ above the electrode structure, which is varied from 0 to $10 \mathrm{~mm}$. Additionally, a water layer thickness of $200 \mathrm{~mm}$ is simulated to determine the asymptotic capacitance for the evaluation of the penetration depth $\gamma_{97 \%}$. The relative permittivity $\varepsilon_{\mathrm{r}}$ is assumed to equal 1 for air, 80 for water and 4.5 for FR4, which is a common material for PCBs. The remaining parameters are varied and therefore described in the corresponding subsections.

\section{A. Number of digits}

The first parameter to be varied is the number of digits $n$ from 3 to 50 , to evaluate its influence on the structure's characteristics. As the electrode area is fixed at $50 \mathrm{~mm} \times 50 \mathrm{~mm}$, a variation of $n$ at the same time means a variation of the spatial wavelength $\lambda$ (see Eq. (2)) from around $18 \mathrm{~mm}$ to $1 \mathrm{~mm}$. For the study of $n$, the substrate thickness $t_{\mathrm{s}}$ equals $1.5 \mathrm{~mm}$ and the electrode thickness $t_{\mathrm{e}}$ equals $36 \mu \mathrm{m}$, which are common values for PCBs. Moreover, the backplane and the coating, depicted in Fig. 1, are neglected and evaluated later in this paper.

The result for the effect of varying $n$ on the capacitance against water layer thickness is shown in Fig. 2. Since the simulations are carried out in two dimensions, the capacitance is given in $\mathrm{pF} / \mathrm{mm}$ and would have to be multiplied with the length of the electrodes $(50 \mathrm{~mm})$ to get the approximated overall capacitance (three dimensions). However, for the evaluation of the effect, we restrict the simulations to two dimensions.
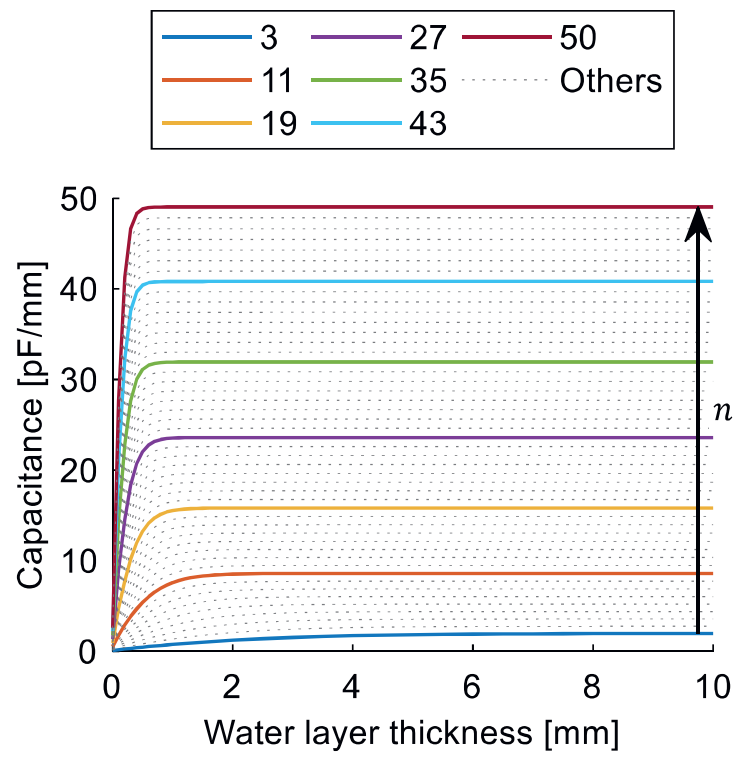

Fig. 2: Capacitance against water layer thickness for a variation of the number of digits $n$ from 3 to 50 .

As Fig. 2 shows, the signal strength increases with the number of digits $n$. While the capacitance of 3 digits (10 $\mathrm{mm}$ water layer thickness) equals around $1.96 \mathrm{pF} / \mathrm{mm}$, the capacitance of 50 digits with around $49 \mathrm{pF} / \mathrm{mm}$ is 25 times greater. Besides the signal strength, also the measurement sensitivity (see Eq. (4)) increases, especially in close range, as the electrodes are moving together and the electric field gets more intensive in this range.

In Fig. 3 the penetration depth and the dynamic range are shown against the number of digits. By increasing $n$, the penetration depth decreases while the dynamic range increases. Since more digits lead to lower electrode gaps, the electric field lines pass a smaller distance and the electric field strength decreases with the height. Thus, the penetration depth declines from 
around $6.8 \mathrm{~mm}$ to around $0.4 \mathrm{~mm}$. In contrast to the penetration depth, the dynamic range increases with the number of digits from approximately $1.9 \mathrm{pF} / \mathrm{mm}$ to $46.4 \mathrm{pF} / \mathrm{mm}$. However, at the same time the basic capacitance increases, too. Since the growth is low in comparison ( $0.09 \mathrm{pF} / \mathrm{mm}$ to $\sim 2.6 \mathrm{pF} / \mathrm{mm}$ ), an improvement of the signal-to-noise ratio can be achieved by increasing the number of digits.

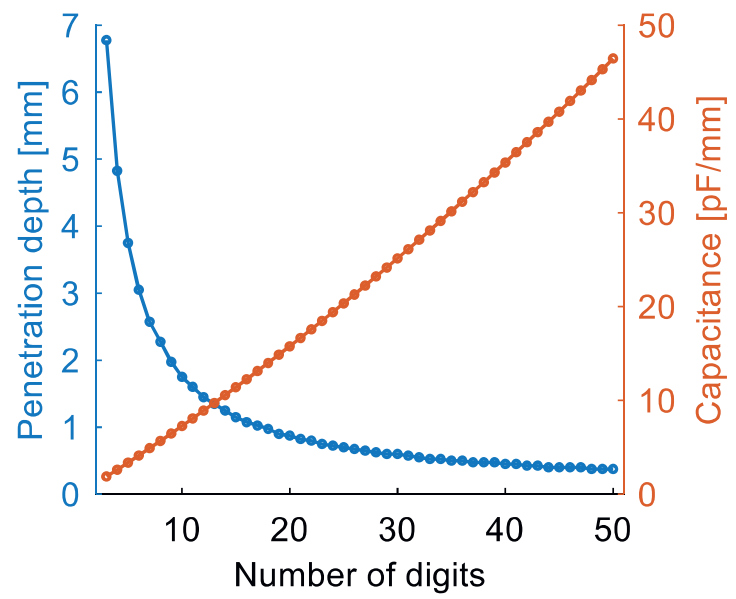

Fig. 3: $\quad$ Penetration depth and dynamic range against number of digits $n$.

By means of the electric field line and potential distribution the significant area around the interdigital electrode structure can be visualized. In Fig. 4 a part of the structure for 25 digits, a water layer thickness of $1 \mathrm{~mm}$ and a substrate thickness of $1.5 \mathrm{~mm}$ is exemplarily shown.

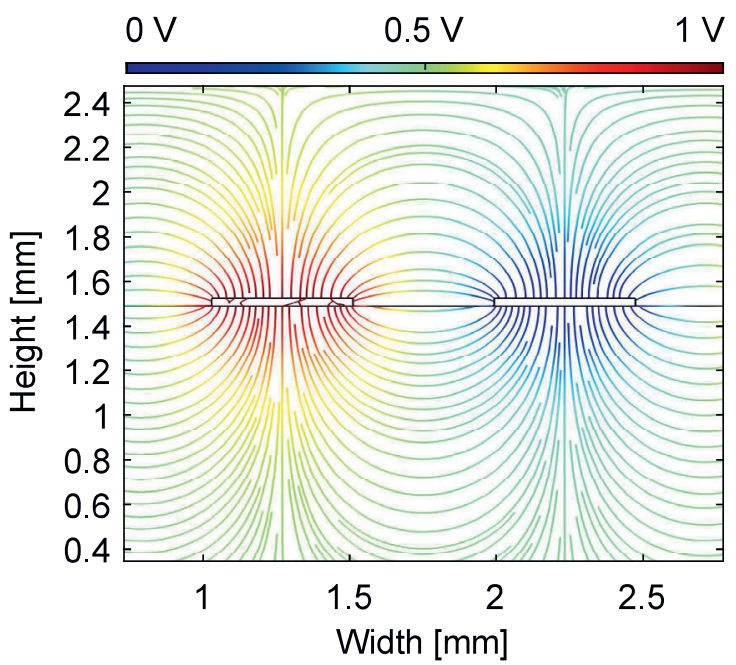

Fig. 4: $\quad$ Electric field (streamlines) and potential distribution (color) for 25 digits.

The electric potential distribution is described by color coding. While blue represents $0 \mathrm{~V}$ and red $1 \mathrm{~V}$, green represents the part of the electric field which is not altered by the electrodes. As illustrated, the electric field penetrates into the medium and the substrate leading both to contribute to the capacitance. Furthermore, the figure enables the penetration depth, which equals $0.7 \mathrm{~mm}$ for 25 digits, to be displayed.

The results demonstrate, there is always a tradeoff between penetration depth and dynamic range or measurement sensitivity when designing an interdigital electrode structure considering the number of digits, respectively the spatial wavelength. Thus, the design parameters have to be adjusted to the application's requirements.

\section{B. Electrode width}

The next parameter to be studied is the electrode width $w$ and hence, the ratio of width $w$ and distance $d$. Therefore, we introduce the dimensionless variable $r$, which is used to calculate the electrode width and the electrode distance depending on the electrode area's width $a$ for the simulations

$$
w=r \cdot \frac{a}{4 n-1}, d=\frac{a-(2 n w)}{2 n-1} .
$$

In order to evaluate the electrode width's effect on the electrode structure's characteristics we exemplarily present results for 25 digits and vary $r$ from 0.3 to 1.7 . In this case 0.3 yields to an electrode width of approximately $0.15 \mathrm{~mm}$ and 1.7 to a distance of around $0.15 \mathrm{~mm}$, which are commonly the minimum track width and the minimum spacing at PCB manufacturers. As for the study of the number of digits, we assume a substrate thickness $t_{\mathrm{s}}$ of $1.5 \mathrm{~mm}$, an electrode thickness $t_{\mathrm{e}}$ of $36 \mu \mathrm{m}$ and neglect backplane and coating.

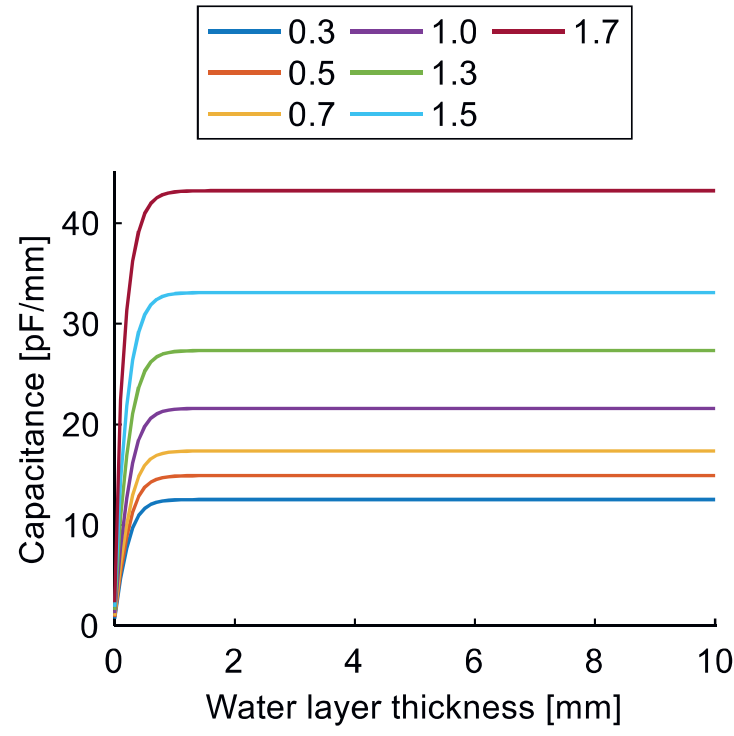

Fig. 5: $\quad$ Capacitance against water layer thickness for a variation of $r$ from 0.3 to 1.7.

The resulting capacitance against water layer thickness curves for a selection of $r$ are shown 
in Fig. 5. All curves show a similar exponential behavior. Increasing $r$ results in a larger signal strength, which ranges from approximately $12.5 \mathrm{pF} / \mathrm{mm}$ in saturation (0.3) to $43.2 \mathrm{pF} / \mathrm{mm}$ (1.7) due to the broader electrodes moving together. As additionally illustrated in Fig. 6, not only the capacitance in saturation increases, but also the dynamic range by more than three times from around $11.8 \mathrm{pF} / \mathrm{mm}$ to $40.9 \mathrm{pF} / \mathrm{mm}$, since the basic capacitance has comparably small values. As a result, the signalto-noise ratio can be extended.

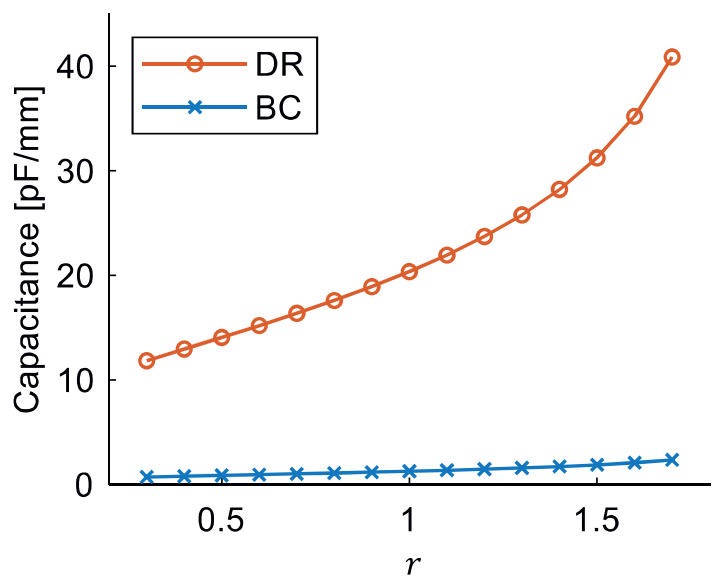

Fig. 6: Dynamic range and basic capacitance against $r$.

\section{Substrate thickness}

In this section, the effect of varying the substrate thickness $t_{\mathrm{s}}$ is studied. For this purpose, we exemplarily present results for an interdigital electrode structure with 25 digits and a varying substrate thickness from $0.1 \mathrm{~mm}$ to $1.5 \mathrm{~mm}$. The electrode thickness $t_{\mathrm{e}}$ is assumed to be $36 \mu \mathrm{m}$ and a coating is neglected. In the first part of the study, we also neglect the backplane depicted in Fig. 1.

An increase in substrate thickness results in a higher signal strength in saturation of about $0.5 \mathrm{pF} / \mathrm{mm}$ for the whole range. It is caused by the electric field that penetrates into a substrate with a permittivity of 4.5 instead of air with a permittivity of 1 . A comparable effect would be caused by changing the substrate's material and thereby the permittivity of it. As Fig. 7 illustrates, the basic capacitance increases with the substrate thickness by a similar value as the signal strength. Thus, the dynamic range, and also the measurement sensitivity, is almost unaffected and the signal-to-noise ratio declines. The basic capacitance increases, but the maximum value is limited due to the penetration depth of the interdigital electrode structure, which is constant (in this instance at $0.7 \mathrm{~mm}$ ) for all substrate thicknesses.

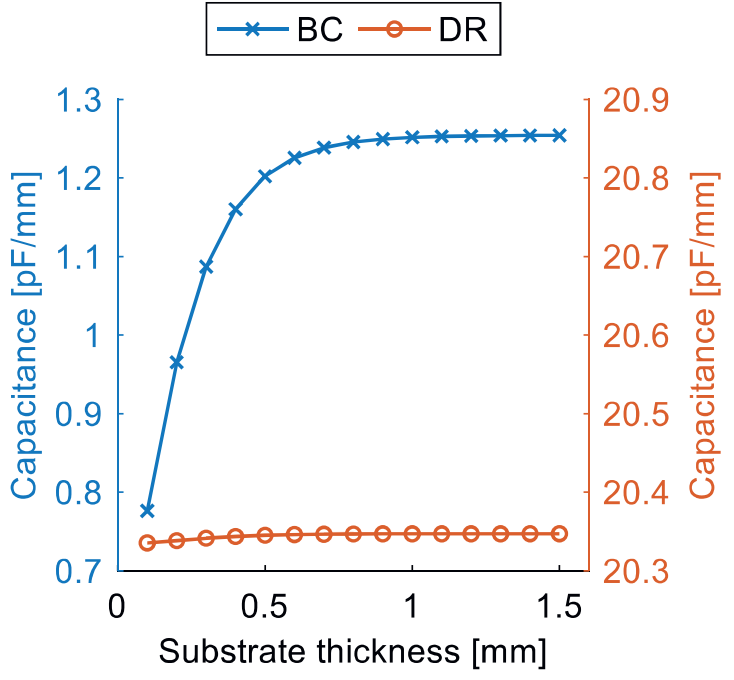

Fig. 7: Dynamic range and basic capacitance against substrate thickness.

In Fig. 8 the electric field line and potential distribution for a part of the structure is shown for a substrate thickness of $0.1 \mathrm{~mm}$ and a water layer thickness of $1 \mathrm{~mm}$. The electric field penetrates through the substrate in the air on the backside. Therefore, not only the monitored medium on the frontside is in the focus of the measurement, but also the medium on the backside, which could lead to misinterpretations and in the context of road wetness detection to fatal errors. Thus, it is preferable to have a substrate thickness thicker than the electrode structure's penetration depth, even though the basic capacitance rises.

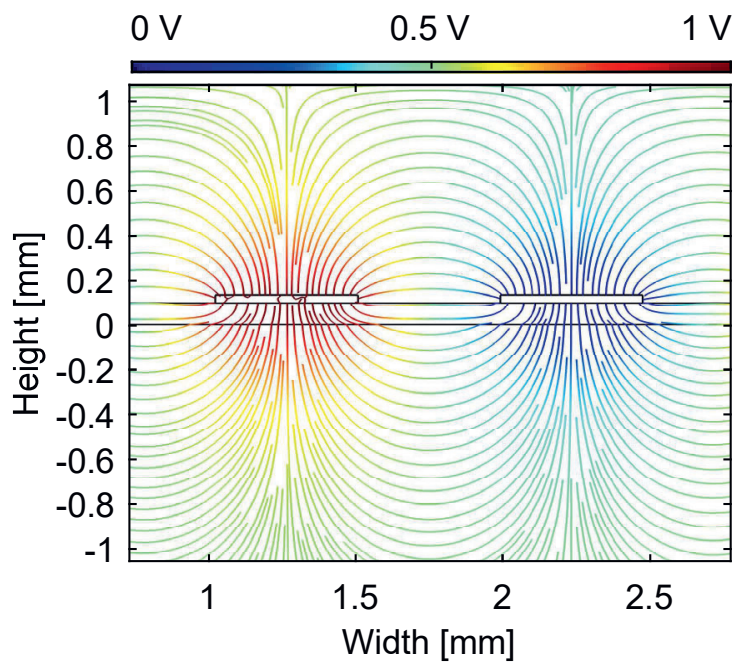

Fig. 8: Electric field (streamlines) and potential distribution (color) for 25 digits and a substrate thickness of $0.1 \mathrm{~mm}$.

Another possibility to limit the measuring range to the frontside, is to use a backplane which shields the electrode structure from noise. In order to evaluate the effect of the backplane, we repeat the simulations with the same settings. In 
contrast to the results without a backplane, a decrease in the substrate thickness leads to a higher signal strength in saturation. The increase in signal strength from a substrate thickness of $1.5 \mathrm{~mm}$ to $0.1 \mathrm{~mm}$ is approximately $6.7 \mathrm{pF} / \mathrm{mm}$ and is thus in a different order of magnitude compared to the simulations without a shield due to the capacitance built between the electrode structure and the backplane. For the same reason, the basic capacitance rises with a decreasing substrate thickness, which is shown in Fig. 9. Besides the basic capacitance, the dynamic range and the measurement sensitivity also increase with reducing the substrate thickness.

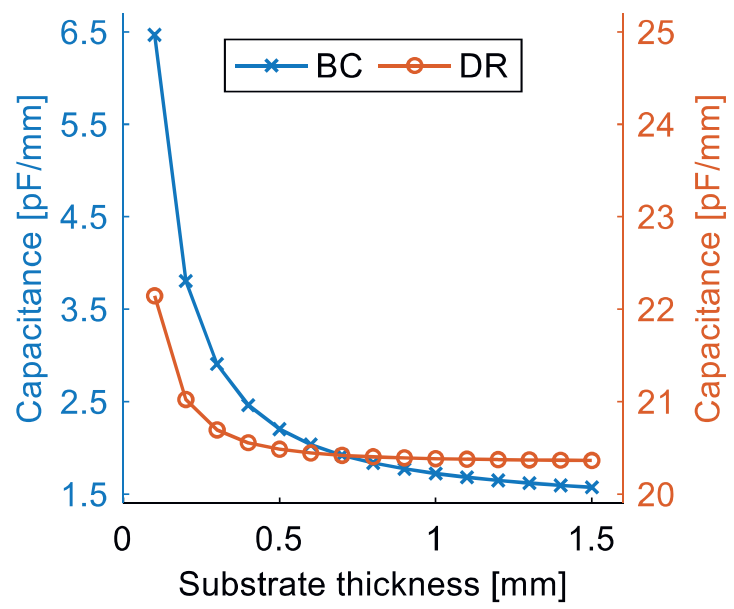

Fig. 9: $\quad$ Dynamic range and basic capacitance against substrate thickness with a backplane.

The electric field line and potential distribution for a part of the studied structure is shown for a substrate thickness of $0.1 \mathrm{~mm}$ and a water layer thickness of $1 \mathrm{~mm}$ in Fig. 10.

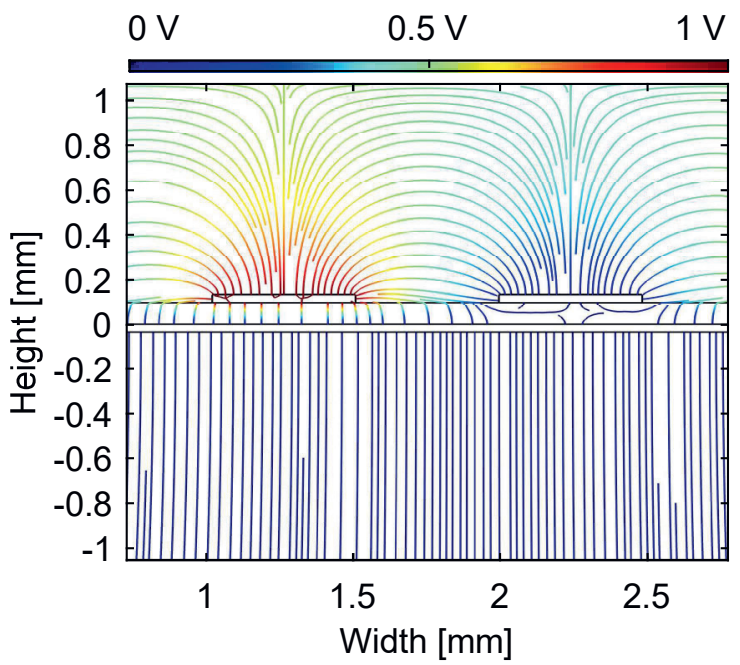

Fig. 10: Electric field (streamlines) and potential distribution (color) for 25 digits and a substrate thickness of $0.1 \mathrm{~mm}$ with a backplane.
In contrast to Fig. 8, the electric field lines cannot penetrate through the substrate due to the backplane. Therefore, only the electrode structure's frontside is relevant for the measurement. The positive electrode and the grounded backplane build a kind of parallel-plate capacitor which results in a high basic capacitance.

\section{Coating}

In this section, we evaluate the effect of the thickness and the permittivity of a coating on top of an interdigital electrode structure. For this purpose, the coating thickness $t_{c}$ is varied from 0 to $1 \mathrm{~mm}$. Additionally, we study the effect of the coating's permittivity by evaluating two materials. For the simulations, a substrate thickness $t_{\mathrm{s}}$ of $1.5 \mathrm{~mm}$ and an electrode thickness $t_{\mathrm{e}}$ of $36 \mu \mathrm{m}$ are assumed. Additionally, the backplane is neglected. In the following we exemplarily present the results for 25 digits.

In Fig. 11, the simulated capacitance against water layer thickness for a variation of coating thickness and a relative permittivity of 3.4 (e.g. polyimide) is presented. As the figure shows, the capacitance is damped increasingly with the coating thickness. Up to a certain coating layer thickness, the course of the capacitance curves shows a similar exponential behavior as before. However, if the coating thickness approaches the structure's penetration depth, the curves get almost constant.

$-0 \mathrm{~mm} \quad-0.1 \mathrm{~mm} \cdots 1.0 \mathrm{~mm}$
$-0.036 \mathrm{~mm}-0.2 \mathrm{~mm}$
$-0.050 \mathrm{~mm}-0.5 \mathrm{~mm}$

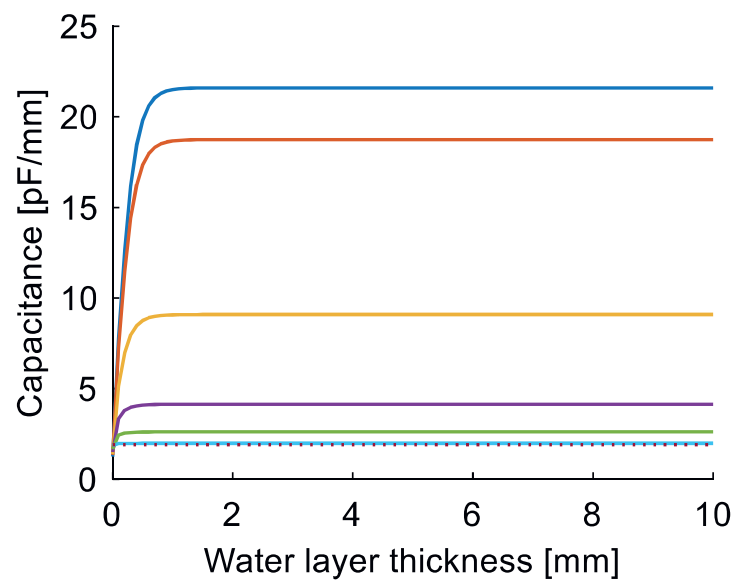

Fig. 11: Capacitance against water layer thickness for a variation of the coating thickness from 0 to $1 \mathrm{~mm}$ and a relative permittivity of 3.4 .

Fig. 12 illustrates the electric field line and potential distribution for a coating thickness of $0.1 \mathrm{~mm}$. In contrast to the behavior in Fig. 8, where no coating is applied, the electric field strength in 
the coating layer is significantly higher than at the transition to the medium. Thus, the coating accounts for a large part of the capacitance, as more field lines pass through it.

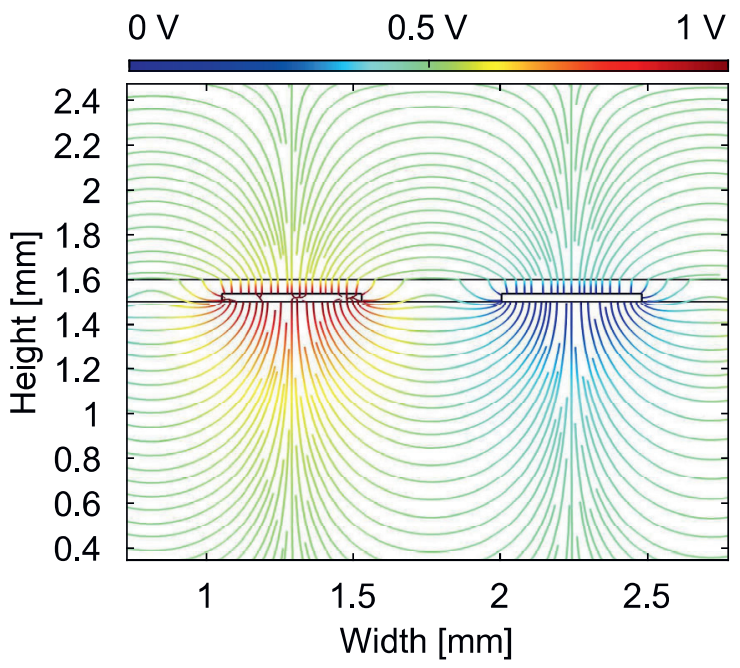

Fig. 12: Electric field (streamlines) and potential distribution (color) for 25 digits and a coating thickness of $0.1 \mathrm{~mm}$ and a relative permittivity of 3.4 .

By increasing the permittivity of the coating layer to 11.7 (e.g. silicon), the behavior alters, as shown in Fig. 13. As the difference in permittivity between the coating and the medium reduces, more electric field lines pass through the coating into the medium resulting in a lower damping of the capacitance. Comparing Fig. 12 and Fig. 13, which represent the same coating thickness, the significant part of the electric field lines penetrating into the medium is noticeably larger.

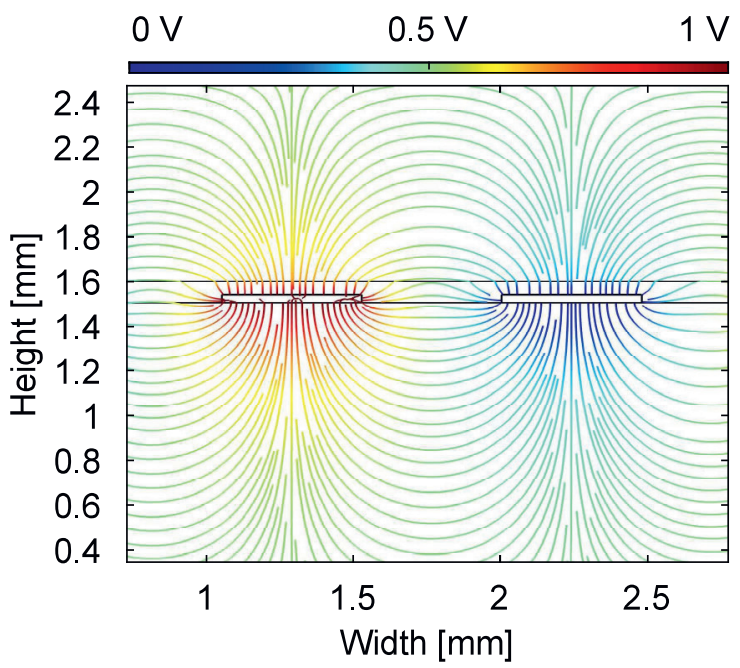

Fig. 13: Electric field (streamlines) and potential distribution (color) for 25 digits and a coating thickness of $0.1 \mathrm{~mm}$ and a relative permittivity of 11.7 .
Fig. 14 shows the dynamic range and basic capacitance against coating thickness for two materials. Due to more electric field lines passing the coating layer, increasing the thickness results in a higher basic capacitance. Furthermore, increasing the permittivity shows the same effect. Similar to the results for the substrate thickness, the maximum basic capacitance is limited due to the penetration depth. By increasing the coating's thickness, the dynamic range decreases significantly in both cases. While there is no difference between the two materials for a thickness of $36 \mu \mathrm{m}$, as the electrodes are not covered on top, a significant variation is noticeable already with a thickness of $50 \mu \mathrm{m}$. Here, the percentage decrease in dynamic range for a relative permittivity of 3.4 is about $62 \%$ and for 11.7 about $38 \%$. Already at $0.1 \mathrm{~mm}$, there is only $13 \%$ of the dynamic range left for a relative permittivity of 3.4 , while there are $32 \%$ left for 11.7. Thus, both curves for the dynamic range converge to zero at different rates showing advantages for a higher relative permittivity.

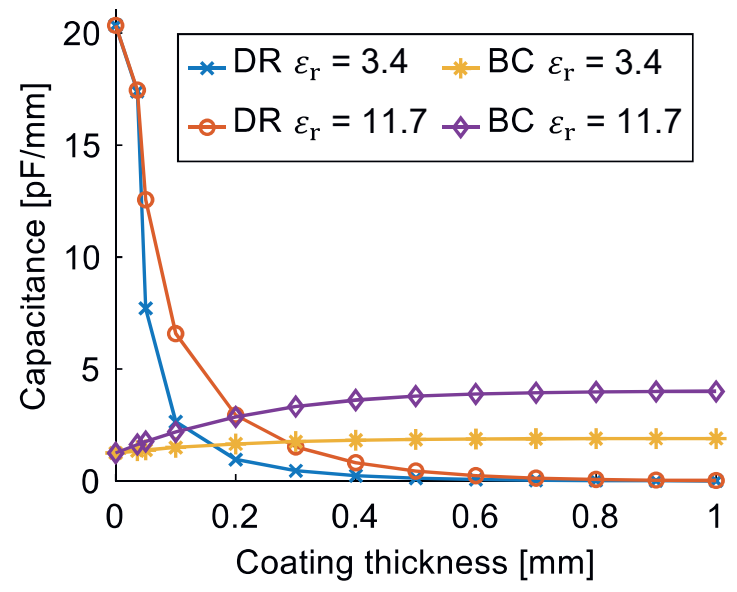

Fig. 14: Dynamic range and basic capacitance against coating thickness.

\section{E. Water spray}

In contrast to the previous simulations, where a static water layer is assumed to simplify the simulations and compare the structure's characteristics, in this section, the structure's behavior towards the presence of water drops is presented. For this purpose, two designs ( 7 and 25 digits) are exemplarily compared in three-dimensional electrostatic simulations. With regard to the results of the previous sections, both designs have an electrode distance of $0.15 \mathrm{~mm}$, a substrate thickness of $1.5 \mathrm{~mm}$ and a backplane. The water drops are realized by hemispheres of three different radii $(0.5 \mathrm{~mm}, 1 \mathrm{~mm}$ and $2.5 \mathrm{~mm})$ and are placed as arrays of size $1 \times 1$ to $5 \times 5$ on top of the electrode structures. 


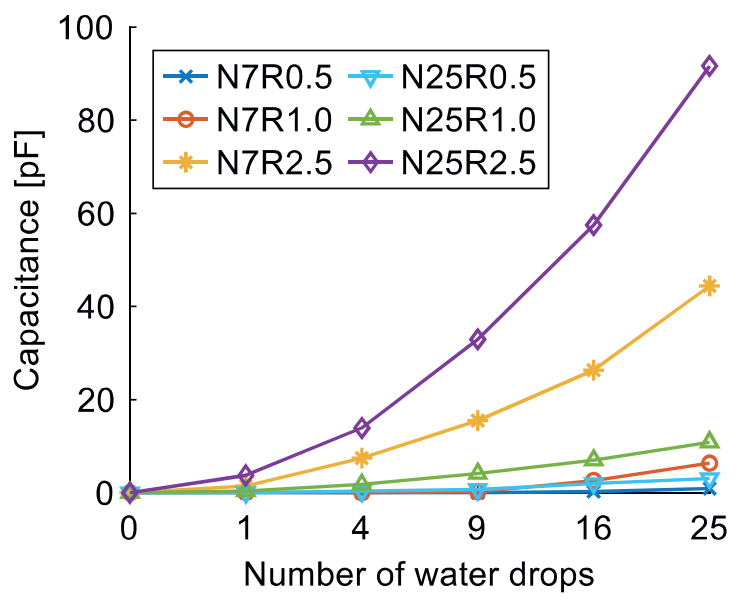

Fig. 15: Capacitance range against number of water drops for two designs.

In Fig. 15, the capacitance range against the number of water drops curves are shown for the two designs. As for the simulations with a static water layer, the increase in capacitance and the measurement sensitivity are higher for a greater number of digits. Especially for smaller drops and low amounts, the design with 25 digits is advantageous, as the increase is considerably greater. The results show the possibility of detecting even small amounts of water drops and confirm the assumptions made in the previous sections.

\section{Conclusions}

This paper presented a study on interdigital electrode structures suitable for the detection of water spray. By means of FEM simulations in COMSOL Multiphysics the structures were analyzed with regard to the effect of varying their design parameters. A variation of the number of digits on a fixed electrode area shows a trade-off between dynamic range and penetration depth. Furthermore, an improvement for the signal-tonoise ratio is possible, as also for increasing the ratio of electrode width to distance. The choice of substrate thickness is directly related to the required penetration depth and a possible shield electrode. In addition, the effect of coating thickness and material were evaluated. Here, the capacitance is damped increasingly with the thickness. By increasing the coating's relative permittivity, the attenuation decreases. Finally, we presented results for the behavior to the presence of water drops and confirmed the assumptions made before.

\section{References}

[1] Statistisches Bundesamt, "Verkehrsunfälle 2017," Verkehr, 2018.

[2] J. Döring, L. Tharmakularajah, J. Happel and K.-L. Krieger, "A novel approach for road surface wetness detection with planar capacitive sensors," Journal of Sensors and Sensor Systems, vol. 8, no. 1, pp. 57-66, 2019, doi: 10.5194/jsss-8-57-2019.

[3] X. Hu and W. Yang, "Planar capacitive sensors - designs and applications," Sensor Review Vol. 30 Issue: 1, pp. 24-39, 2010, doi: 10.1108/02602281011010772.

[4] L. K. Baxter, Capacitive Sensors: Design and Applications, New-York: Wiley-IEEE Press, 1997, doi: 10.1109/9780470544228.

[5] G. Brasseur, "Design rules for robust capacitive sensors," IEEE Transactions on Instrumentation and Measurement, vol. 52, no. 4 , pp. 1261-1265, 2003, doi: 10.1109/TIM.2003.816812.

[6] H. Canbolat, "A Novel Level Measurement Technique Using Three Capacitive Sensors for Liquids," IEEE Transactions on Instrumentation and Measurement, vol. 58, no. 10 , pp. $3762-3768,2009$, doi: 10.1109/TIM.2009.2019715.

[7] J. Happel, J. Döring, K.-L. Krieger, J. Deitschun and D. Godlinski, "Printed Capacitive Sensors for Contactless Ice Detection in Automotive Liquid Conveyor Pipes," AMA Conferences -- Proceedings Sensor, pp. 127-132, 2017, doi: 10.5162/sensor2017/P3.2.

[8] J. Mizuguchi, J. C. Piai, J. A. d. França, M. B. d. M. França, K. Yamashita and L. C. Mathias, "Fringing Field Capacitive Sensor for Measuring Soil Water Content: Design, Manufacture, and Testing," IEEE

Transactions on Instrumentation and Measurement, pp. 212-220, January 2015, doi: 10.1109/TIM.2014.2335911.

[9] M. J. da Silva, Impedance Sensors for Fast Multiphase Flow, Technical University of Dresden, 2008

[10] A. V. Mamishev, K. Sundara-Rajan, F. Yang, Y. Du and M. Zahn, "Interdigital sensors and transducers," Proceedings of the IEEE 92, pp. 808-845, May 2004, doi: 10.1109/JPROC.2004.826603.

[11] R. N. Dean, A. K. Rane, M. E. Baginski, J. Richard, Z. Hartzog and D. J. Elton, "A Capacitive Fringing Field Sensor Design for Moisture Measurement Based on Printed Circuit Board Technology," IEEE Transactions on Instrumentation and Measurement, vol. 61 , no. 4, pp. $1105-$ 1112, April 2012, doi: 10.1109/TIM.2011.2173041.

[12] X. B. Li, S. D. Larson, A. S. Zyuzin and A. V. Mamishev, "Design principles for multichannel fringing electric field sensors," IEEE Sensors Journal, vol. 6, no. 2, pp. 434-440, 2006, doi: 10.1109/JSEN.2006.870161. 